\title{
Investissement et diversification économique au Gabon
}

\author{
Paterne Ndjambou, Doctorant en développement régional \\ Université du Québec à Chicoutimi
}

\section{INTRODUCTION}

\section{Le chemin pour atteindre le développement durable en Afrique est long et semé d'embûches à cause de sa forte dépendance à \\ l'exploitation et exportation des ressources naturelles, source de détérioration de \\ l'écosystème et menace de la biodiversité.}

Une économie forte, croissante et durable est l'objectif visé par toute nation du monde. En effet, le développement durable est censé améliorer les conditions de vies des populations par la création de l'emploi et par le progrès du développement humain. Or, le chemin pour atteindre le développement durable en Afrique est long et semé d'embûches à cause de sa forte dépendance à l'exploitation et exportation des ressources naturelles, source de détérioration de l'écosystème et menace de la biodiversité. Selon plusieurs auteurs, la diversification économique est l'un des leviers qui permettrait d'atteindre les objectifs du développement durable ${ }^{1}$.

Culturellement diversifié, faiblement peuplé, politiquement stable et richement nanti en ressources naturelles et en biodiversité, le Gabon est un pays de l'Afrique centrale ayant une économie fortement concentrée et dépendante de la rente pétrolière. Le pétrole représentait $59 \%$ des recettes budgétaires et plus de $80 \%$ des recettes d'exportations du pays en $2007^{2}$. La dépendance de l'économie gabonaise au pétrole perdure depuis le milieu des années soixante-dix. La classe politique et l'intelligentsia gabonaises s'accordent pour dire qu'il faut engager sérieusement et rapidement le pays dans une stratégie de diversification économique pour étoffer son tissu économique dans le but de renforcer sa compétitivité internationale et de réduire sa dépendance à l'or noir et sa vulnérabilité aux chocs externes ${ }^{3}$.

Bien que plusieurs études empiriques ${ }^{4}$ aient démontré que l'investissement de capitaux importants est l'un des facteurs explicatifs de la diversification économique dans plusieurs contextes, il n'en reste pas moins qu'aucune étude n'a spécifiquement pas encore été menée sur le sujet au Gabon. C'est dans cette perspective que cet article propose une analyse de l'impact exercé par l'ensemble des investissements réalisés sur le taux de diversification économique au Gabon durant les trois dernières décennies.

À cette fin, la première section précise les liens théoriques et empiriques entre investissement et diversification économique. La deuxième partie présente les cadres conceptuel et méthodologique de la recherche. La troisième partie expose les résultats et les discussions en regard des études antérieures et du contexte spécifique $\mathrm{du}$ Gabon. La conclusion fait ressortir les apports de cette étude et suggère quelques pistes d'action qui pourraient inspirer les politiques économiques nationales $\mathrm{du}$ gouvernement gabonais.

Culturellement diversifié, faiblement peuplé, politiquement stable et richement nanti en ressources naturelles et en biodiversité, le Gabon est un pays de l'Afrique centrale ayant une économie fortement concentrée et dépendante de la rente pétrolière. Le pétrole représentait $59 \%$ des recettes budgétaires et plus de $80 \%$ des recettes d'exportations du pays. 


\section{LE LIEN ENTRE INVESTISSEMENT ET DIVERSIFICATION ÉCONOMIQUE}

\subsection{Théorie «Éclectique» de Dunning}

Le modèle (Ownership-LocalizationInternalization) stipule qu'une multinationale compétitive est une entreprise qui détient un avantage spécifique " Ownership advantage ", qui bénéficie d'un avantage de localisation "Localization advantage » et qui profite d'un avantage d'internalisation «Internalization advantage ".

Le paradigme «Éclectique» est actuellement l'une des explications les plus satisfaisantes pour interpréter la dynamique des multinationales à l'ère de la mondialisation. Le modèle de Dunning $^{5}$ stipule que les stratégies d'investissement des entreprises multinationales sont liées à la combinaison des avantages spécifiques à la firme (capital intellectuel, capacité d'innovation, savoir-faire, capacité stratégique), aux avantages offerts par des pays-hôtes (ressources humaines et naturelles, coût en énergie, fiscalité, collaboration gouvernementale) et aux avantages de l'internalisation (coût de transaction, risque de transfert des connaissances). Autrement dit, le modèle (OwnershipLocalization-Internalization) stipule qu'une multinationale compétitive est une entreprise qui détient un avantage spécifique "Ownership advantage ", qui bénéficie d'un avantage de localisation «Localization advantage » et qui profite d'un avantage d'internalisation « Internalization advantage $»$.

L'un des leviers de l'internalisation des multinationales est l'investissement direct à l'étranger (IDE) qui leur offre, entre autres, l'opportunité d'acquérir des nouveaux marchés. Pour les payshôtes, qu'ils soient développés ou en développement, il facilite l'accessibilité aux transferts technologiques et au savoir-faire managérial, ce qui peut se traduire à terme par des gains de productivité dans les entreprises locales. Une étude menée sur neufs pays de l'Europe centrale et australe montre empiriquement qu'il y a une relation positive entre l'IDE et la diversification des exportations dans ces pays ${ }^{6}$. Cette étude conclut également que l'IDE influe sur le degré de sophistication des produits exportés. Dans les buts d'améliorer l'attractivité des multinationales, les gouvernements doivent investir dans une infrastructure publique de qualité, développer le capital humain et pratiquer une politique macroéconomique renforçant la stabilité politique, source d'encouragement des investissements sur le long terme ${ }^{7}$. L'idée de l'investissement public comme facteur facilitateur de la diversification économique a également été étudiée dans la théorie de la croissance endogène qu'il convient de préciser.

\subsection{Théorie de la croissance endogène}

La théorie de la croissance endogène reconnait l'intervention de l'État dans l'investissement dans la recherche et développement (R\&D), dans la fourniture des biens publics (infrastructure) et dans la régularisation du marché en contexte de concurrence imparfaite comme des leviers de la croissance économique d'une entité géographique quelconque. C'est en vertu de cette théorie que l'investissement public organisé par un «État développementiste » dans les infrastructures et les secteurs productifs ciblés a un impact sur la diversification économique et sur le taux de croissance du pays à long terme ${ }^{8}$. C'est en intégrant la diversification économique comme l'un des facteurs de la croissance économique que Romer ${ }^{9}$ a fourni un argument supplémentaire à sa perspective de la théorie de la croissance endogène. En effet, selon cet auteur, la diversification des intrants (biens intermédiaires) dans la production peut être bénéfique pour la croissance. Adaptant cet argument de la diversification des intrants à la diversification de la production, d'autres chercheurs ${ }^{10}$ sont arrivés aux mêmes conclusions dans le cas de la Corée du Sud et de Taïwan. D'autres études empiriques ${ }^{11}$ ont démontré à partir des méthodologies différentes, qu'un pays ou une région économiquement diversifié est moins sensible aux aléas conjoncturels, et ce, à condition que les aléas qui touchent les différents secteurs d'activités ne soient pas positivement bien corrélés. 


\section{CADRE CONCEPTUEL ET MÉTHODOLOGIQUE DE LA RECHERCHE}

\subsection{Cadre conceptuel et hypothèses}

L'investissement public est constitué par l'ensemble des dépenses engagées par l'État et les collectivités territoriales en équipement collectif ou infrastructures publiques : routes, aéroports, chemin de fer, hôpitaux, écoles, logements sociaux, etc.

Le modèle de recherche d'analyse illustré à la figure 1 met en relief l'investissement et la diversification économique. L'investissement est analysé à partir de deux construits théoriques à savoir l'investissement public (IP) et l'investissement direct à l'étranger (IDE). La diversification économique est, quant à elle, analysée à l'aide de l'Indice de Hirschman Normalisé (IHN).

L'investissement public est constitué par l'ensemble des dépenses engagées par l'État et les collectivités territoriales en équipement collectif ou infrastructures publiques: routes, aéroports, chemin de fer, hôpitaux, écoles, logements sociaux, etc. À l'instar des études cihaut citées, la Formation Brute du Capital Fixe (FBCF) est utilisée comme indicateur de mesure de l'investissement public au Gabon. Pour ce faire, il sied bien de recourir aux dépenses d'investissement dans le secteur productif, l'infrastructure, les secteurs sociaux qui se traduisent généralement par une modification de la valeur $\mathrm{du}$ patrimoine du pays et des collectivités territoriales.

L'investissement direct à l'étranger (IDE) est une opération par laquelle un agent économique acquiert des actions ou des parts de propriété dans une entreprise d'un autre pays dans le but d'exercer une influence sur la gestion et la prise de décision. Pour le Fonds Monétaire International $(\mathrm{FMI})^{12}$, l'IDE peut prendre quatre formes: la création d'une entreprise ou d'un établissement à l'étranger, l'acquisition d'au moins $10 \%$ du capital social d'une entreprise étrangère déjà existante, le réinvestissement de ses bénéfices par une filiale ou une succursale située à l'étranger et les opérations entre maison mère d'une multinationale et ses filiales (prêts, avances de fonds, etc.). Pour les fins de cette recherche, la nomenclature du FMI à partir de laquelle la CNUCED s'inspire pour élaborer ses statistiques a été retenue.

\section{L'investissement direct à l'étranger (IDE) est une opération par laquelle un agent économique acquiert des actions ou des parts de propriété dans une entreprise d'un autre pays dans le but d'exercer une influence sur la gestion et la prise de décision.}

La diversification économique est le processus de transformation structurelle d'une économie qui migre d'un tissu dominé par les secteurs d'activités primaires (ressources naturelles, agriculture, etc.) vers les secteurs secondaires (industries, manufactures, etc.) et tertiaires (services, tourisme, etc.) ${ }^{13}$. En d'autres mots, la diversification économique est le processus de densification du tissu économique d'une entité géographique quelconque (pays, collectivité locale, etc.) grâce à la création des nouveaux secteurs d'activités ou à l'expansion des secteurs d'activités déjà existants.

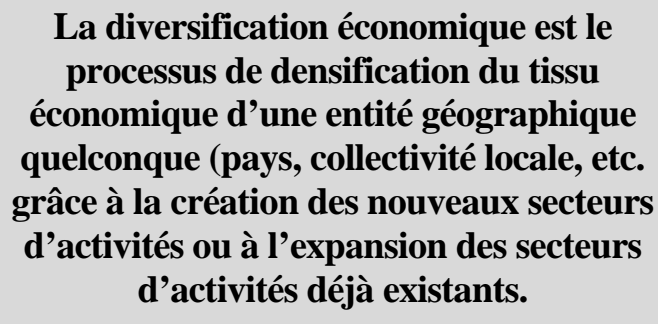

Tel qu'énoncé précédemment, l'IHN est l'indicateur de mesure de la diversification économique au Gabon. Cet indice est calculé à l'aide d'une formule mathématique contentant le nombre de produits exportés par le Gabon, la valeur des exportations de chaque produit et la valeur des exportations totales du pays. L'IHN est une valeur comprise entre 0 et 1 . Lorsqu'il est proche de 1, l'économie analysée est considérée comme fortement concentrée, donc peu diversifiée. Par contre, quand il est proche de 0 , l'économie étudiée est considérée comme étant relativement diversifiée. 
Selon le modèle proposé dans le cadre de cette étude, l'investissement global influence significativement la diversification économique au Gabon. Cette proposition générale se traduit par deux arguments spécifiques. D'abord, l'investis- sement public influence significativement la diversification économique au Gabon. Ensuite, l'investissement direct à l'étranger influence significativement la diversification économique au Gabon.

Figure 1 : Modèle d'analyse entre investissement et diversification économique

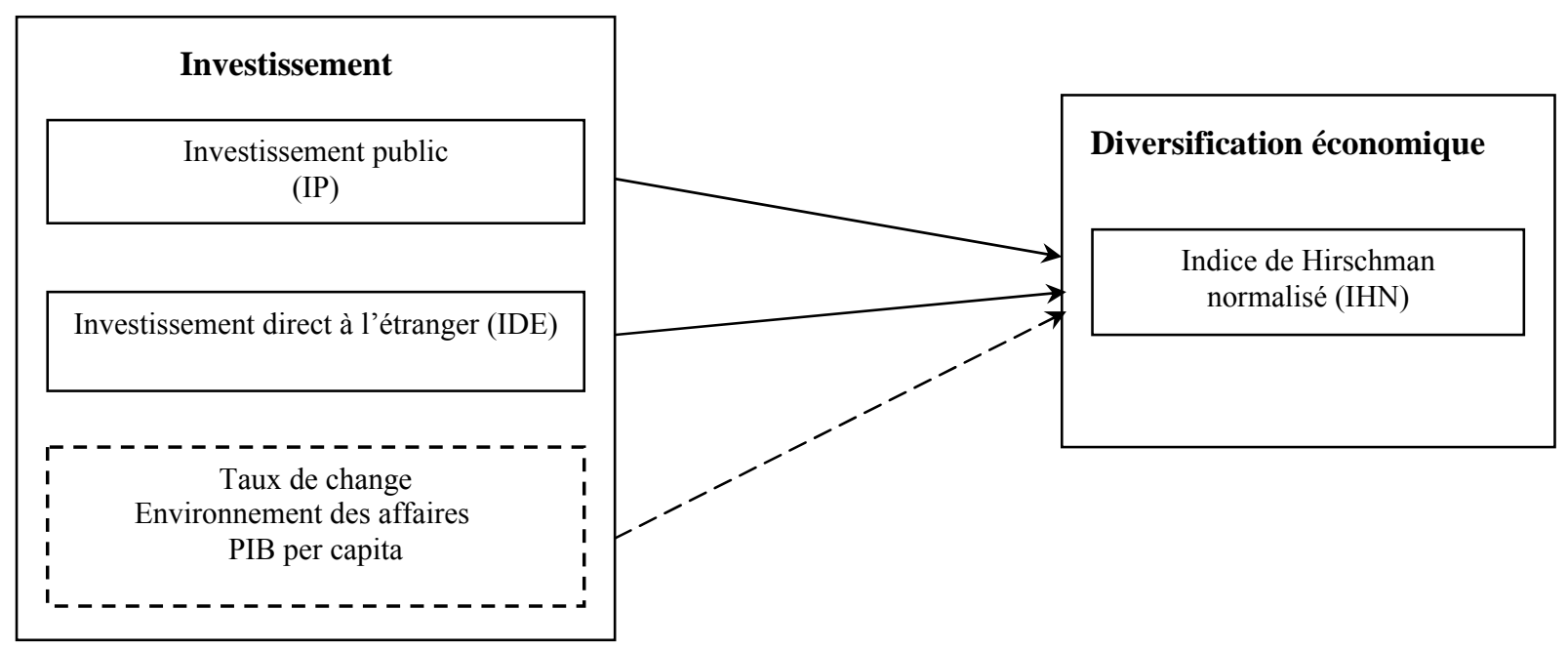

\subsection{Cadre méthodologique}

Le modèle à la base de cette étude a été formalisé par une équation économétrique où la diversification économique dépend de cinq variables explicatives: investissement public, investissement direct à l'étranger, taux de change, environnement des affaires et produit intérieur brut per capita. Mais pour les besoins de cet article, il a été décidé de ne retenir que l'IP et l'IDE comme variables prédictives de la diversification économique au Gabon. Ce choix se justifie par la priorité de vérifier à la fois les effets des stratégies d'investissement du gouvernement et celles des investisseurs étrangers sur le processus de densification du tissu économique du Gabon.

Les données chronologiques annuelles de 1980 à 2008 sur l'IHN, l'IP et l'IDE ont été collectées auprès des trois sources différentes. En ce qui concerne l'IHN, deux sources de données ont été consultées, à savoir la base des données de la Conférence des Nations Unies pour le Com- merce et le Développement (CNUCED) pour la période de 1995 à 2008 et celle de la Commission Économique pour l'Afrique des Nations Unies (UNECA) de 1980 à 1994. Les données sur la formation brute du capital ont été fournies pour l'ensemble de la période d'étude par la Direction Générale du Budget (DGB) du Ministère du Budget, des Comptes publics, de la Fonction publique, chargé de la Réforme de l'État. Enfin, les données sur l'investissement direct à l'étranger ont été recueillies auprès de la CNUCED.

Pour estimer le modèle d'analyse, la méthode des Moindres Carrées Ordinaires (MCO) en régression multiple linéaire a été mise à contribution. Après avoir calculé le coefficient de Pearson (r), les tests statistiques de Fisher, de Student et de Durbin-Watson ont été effectués. Le traitement et l'analyse de données ont été faits à l'aide du progiciel SPSS 18.0 pour Windows. C'est sa fiabilité et sa robustesse qui ont amenés à y avoir recours comme outil de traitement et d'analyse de données. 


\section{RÉSULTATS ET DISCUSSIONS}

\section{Graphique 1: Indice de diversification économique du Gabon}

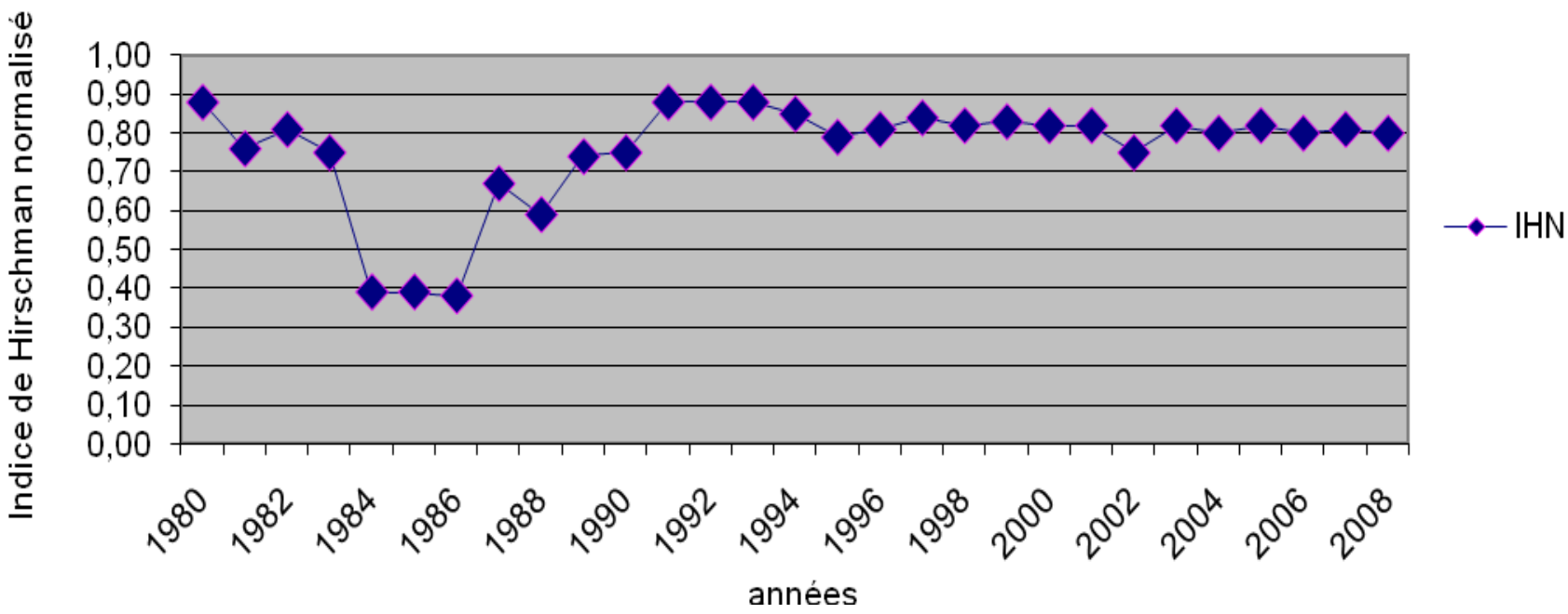

\section{1 Évolution des variables de 1980 à 2008}

Tel que présenté au graphique 1, trois phases caractérisent l'évolution de l'indice de diversification économique du Gabon au cours de la période 1980-2008. De 1980 à 1986, cet indice est passé de 0,88 à 0,38 ce qui signifie que l'économie gabonaise s'est progressivement diversifiée et le nombre et la variété des produits exportés ont augmenté. De 1986 à 1991, la tendance d'évolution de l'indice s'est inversée passant de 0,38 à 0,88 . D'une diversification relative, l'économie gabonaise s'est de nouveau concentrée. Au cours de la période 1991-2008, l'indice de diversification économique du pays est quasiment resté stable dans la zone de forte concentration. Pour résumer, le Gabon a une économie concentrée dans l'exploitation des ressources naturelles et ses principaux produits d'exportation sont le pétrole, le bois et le manganèse.

\section{Pour résumer, le Gabon a une économie concentrée dans l'exploitation des ressources naturelles et ses principaux produits d'exportation sont le pétrole, le bois et le manganèse.}

En matière d'investissement public, le graphique 2 montre qu'au cours de la période 1980-2008, le budget d'investissement ordonnancé et exécuté par l'État gabonais n'a pas été stable. De 1980 à 1986, la FBCF a plus que de doublé passant de 399 à 855 millions de dollars américain. Les pétrodollars engrangés lors des deux boums pétroliers ont prioritairement été investis dans des projets de développement du secteur productif et d'aménagement des infrastructures du pays. Cet effort d'investissement initié par la classe dirigeante n'a malheureusement pas pu être maintenu puisque la FBCF a baissé à 256 millions de dollars en 1988 . Cette baisse drastique est l'une des conséquences de l'effritement des recettes de l'État causé par la crise de la dette qui a fait chuter le cours de l'or noir. Même si le gouvernement a su maintenir un budget d'investissement moyen de 255 millions entre 1988 et 1998; il n'en demeure pas moins que cette moyenne a chuté à 146 millions pour la période de 1999-2003. Ce n'est qu'à partir de 2004 que le budget d'investissement a repris son ascension pour atteindre 665 millions de dollars en 2008. Cette augmentation de la FBCF est surtout attribuable à la hausse des recettes pétrolières dont le cours du Brent a atteint le sommet historique de $145 \$$ en juillet 2008 après avoir débuté son ascension en 2003 avec l'invasion américaine en Irak. 
Graphique 2: Investissement public en millions de \$ US

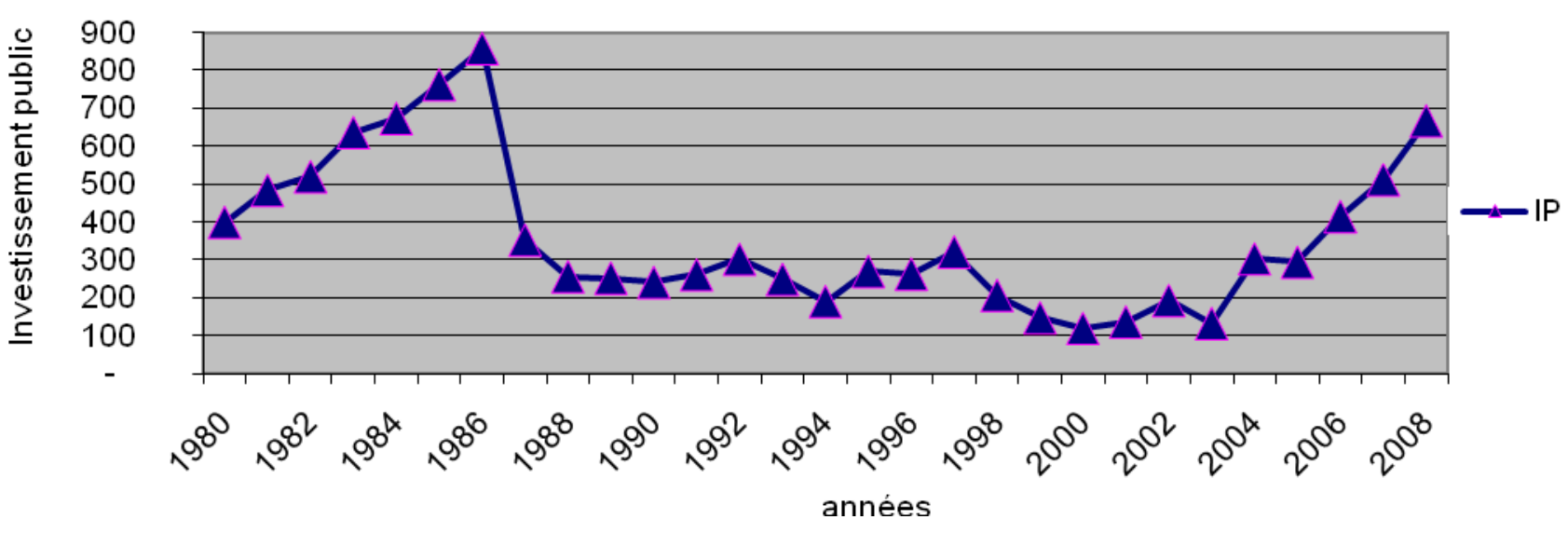

En ce qui a trait à l'IDE au Gabon, le graphique 3 montre que trois phases délimitent l'évolution de cette variable. La première phase, qui correspond à la décennie des années quatre vingt, se singularise par une moyenne annuelle d'IDE de près de 73 millions de dollars américains. Les investisseurs étrangers ont injecté des flux dans l'économie gabonaise sous forme de capital social ou de bénéfices réinvestis. La décennie des années quatre vingt dix correspond à la deuxième phase qui se particularise par un désinvestissement ou une réduction des flux de l'IDE au Gabon avec un moyenne de moins de 43 millions de dollars. La réduction des flux de
l'IDE au Gabon pourrait s'expliquer par le fait que les multinationales œuvrant au Gabon ont rapatrié dans leurs pays d'origine une part des bénéfices réalisés plus grande que celle qu'elles ont réinvestie dans l'économie locale. L'une des raisons de ce rapatriement massif réside dans le fait que les investisseurs étrangers auraient peutêtre pris peur à cause des soubresauts qu'a connus le Gabon au début des années quatre vingt dix avec la conférence nationale réinstaurant la démocratie en 1990 et les émeutes postélectorales de la première élection démocratique du pays en 1993.

\section{Graphique 3: Investissement direct à l'étranger en millions de \$ US}

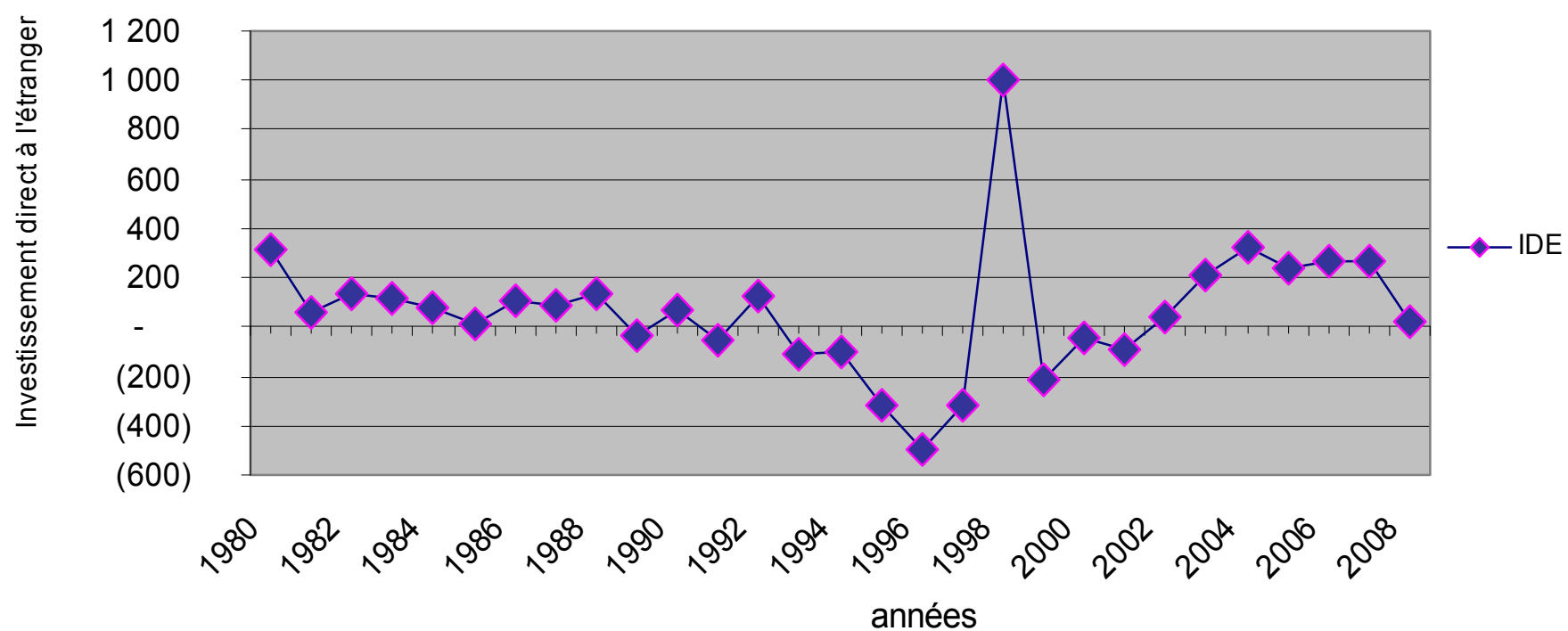


Malgré une moyenne négative des flux d'IDE, il est important de souligner que c'est en 1998 que « la Cendrillon d'Afrique " enregistre un flux d'IDE de près d'un milliard de dollars. La troisième phase qui couvre la décennie des années deux mille se caractérise par des flux d'IDE plus importants avec une moyenne annuelle, durant la période, de près de 154 millions de dollars américains. Le Gabon retrouve de son attractivité pour les investisseurs étrangers. Cette attractivité a été stimulée par des réformes économiques initiées par le gouvernement avec l'adoption de la charte des investissements en 1998, du code minier en 2000 et du code forestier en 2001. Ces réformes ont solidifié les dispositifs internationaux de garantie des investissements pour rassurer les investisseurs étrangers à investir au Gabon.

\subsection{Validation du modèle et discussion}

\section{En effet, les résultats empiriques démontrent que l'investissement global a une influence significative sur la diversification économique en contexte gabonais.}

Tous les tests statistiques effectués valident la robustesse du modèle d'analyse de la diversification économique au Gabon à partir de cinq variables explicatives incluses dans le modèle général. En effet, les résultats empiriques démontrent que l'investissement global a une influence significative sur la diversification économique en contexte gabonais. Il est important d'ajouter que cette influence s'explique non seulement par la contribution des investissements publics, mais aussi par celle des investissements directs à l'étranger.

Tout d'abord, les résultats empiriques de cette recherche montrent que l'investissement public influence significativement la diversification économique au Gabon. Plus particulièrement, une hausse de $1 \%$ de l'investissement public se traduirait par une baisse de 6,30.10-10\% de l'IHN. Puisqu'on sait qu'une baisse de cet indice signifie une plus grande diversification économique, il est donc possible d'affirmer que l'investissement public est un déterminant de la diversification économique au Gabon. Ce résultat valide empiriquement la théorie de la croissance endogène en contexte gabonais et corrobore les résultats de l'étude de la CNUCED antérieurement citée. Par ailleurs, les résultats statistiques confirment aussi que l'investissement direct à l'étranger influence significativement la diversification économique au Gabon. En effet, une augmentation de $1 \%$ de l'IDE s'accompagnerait d'une baisse de 1,37.10-11\% de l'IHN, donc d'une amélioration de la diversification économique du pays. Il appert, à la lumière de ce résultat, que les multinationales contribuent au processus de diversification économique du Gabon à l'instar de ce qu'elles ont fait en Corée du Sud, à Taïwan, en Europe centrale et ailleurs dans le monde.

Enfin, dans la mesure où l'investissement public et l'investissement direct à l'étranger sont des déterminants de la diversification économique au Gabon, force est de constater que leur niveau d'influence n'est pas le même. L'investissement public a plus d'impact sur la diversification économique du pays que l'investissement direct à l'étranger. Cette pondération du niveau d'influence de deux types d'investissement suscite des interrogations relatives aux stratégies d'investissement du gouvernement gabonais et des multinationales œuvrant dans le pays.

\section{L'investissement public a plus d'impact sur la diversification économique du pays que l'investissement direct à l'étranger.}

À la lumière des résultats statistiques et des évolutions de l'investissement public et de l'investissement direct à l'étranger, il ressort cependant qu'un faible niveau d'investissement public accompagné d'une stratégie gouvernementale non structurée pourrait expliquer l'inefficacité de l'investissement public sur la densification du tissu économique du pays. La réduction des flux d'investissement direct à l'étranger au Gabon durant la décennie des années quatre vingt-dix et la tendance des investisseurs étrangers à investir prioritairement dans les secteurs des ressources naturelles sont de nature à favoriser la spécialisation économique plutôt que la diversification. 


\section{CONCLUSION}

Dans le but d'insuffler une dynamique au processus de diversification économique, le gouvernement gabonais devra judicieusement cibler les secteurs dans lesquels investir pour un développement économique et territorial diversifié et viable.

Cette étude corrobore les résultats des travaux qui ont démontré le rôle de l'investissement dans la diversification économique. Elle démontre que l'investissement public et l'investissement direct à l'étranger sont des facteurs explicatifs de la diversification économique au Gabon. Elle relance le débat sur le rôle de l'État dans l'élaboration et la mise en œuvre des politiques pouvant concourir à l'attractivité des territoires et à leur développement. Dans le but d'insuffler une dynamique au processus de diversification économique, le gouvernement gabonais devra judicieusement cibler les secteurs dans lesquels investir pour un développement économique et territorial diversifié et viable.

Contrairement aux stratégies centralisées appliquées jusqu'ici, les autorités gabonaises devront désormais miser sur des stratégies décentralisées par une ingénierie territoriale qui consiste à impliquer toutes les forces vives de la nation, société civile, secteur privé et collectivités locales pour identifier les potentialités intéressantes et définir un projet de territoire susceptible de relancer le processus de diversification économique du pays.

\section{Contrairement aux stratégies centralisées appliquées jusqu'ici, les autorités gabonaises devront désormais miser sur des stratégies décentralisées par une ingénierie territoriale qui consiste à impliquer toutes les forces vives de la nation, société civile, secteur privé et collectivités locales pour identifier les potentialités intéressantes et définir un projet de territoire susceptible de relancer le processus de diversification économique du pays.}

Au chapitre des potentialités, le Gabon n'est pas sans atout à ce niveau puisqu'il dispose des pistes attrayantes pour une diversification à la fois verticale et horizontale de son économie. L'investissement public dans le secteur primaire via la pêche industrielle, l'aquaculture, l'agriculture et l'élevage pourrait contribuer à la diversification horizontale de l'économie. Il pourrait surtout réduire l'insécurité alimentaire du pays dont $40 \%$ des besoins sont couverts par les importations. L'exploitation des nombreuses ressources minières du pays à l'image du fer, de l'or et du diamant contribuerait à réduire la dépendance vis-à-vis de l'exploitation de l'or noir.

\section{L'investissement public dans le secteur} primaire via la pêche industrielle, l'aquaculture, l'agriculture et l'élevage pourrait contribuer à la diversification horizontale de l'économie. Il pourrait surtout réduire l'insécurité alimentaire du pays dont $40 \%$ des besoins sont couverts par les importations.

Dans une perspective de diversification verticale, la forêt gabonaise qui représente $85 \%$ $\mathrm{du}$ territoire offre une possibilité en matière d'industrialisation de la filière bois par la première, deuxième, voire la troisième transformation du bois d'œuvre qui est actuellement exporté sous forme de grumes. Les treize parcs nationaux qui représentent $11 \%$ du territoire offrent des possibilités très intéressantes pour le développement de l'écotourisme à l'ère où le développement durable est au cœur des préoccupations mondiales avec la pression induite par les changements climatiques.

Somme toute, une politique industrielle et sectorielle bien conçue avec une évaluation minutieuse des effets d'entraînement et des effets multiplicateurs anticipés pourrait sans doute à long terme stimuler les investisseurs étrangers à investir dans des secteurs autres que les mines, les hydrocarbures et la foresterie. 


\section{BIBLIOGRAPHIE}

${ }^{1}$ Shediac, R., Abouchakra, R., Moujaes, C.N and Ramsay, M. (2008), Economic Diversification: The road to the sustainable development, Booz \&Company, 16 pages. Pezzey, J. (1992), « Sustainability: an interdisciplinary guide », Environnemental Values $\mathrm{n}^{\mathrm{o}}$ 1, pp. 321-362.

${ }^{2}$ Direction générale de la statistique et des études économiques (2009), Annuaire Statistique du Gabon: 2001-2007, Numéro 11, Ministère du Développement, de la Performance Publique, de la Prospective et de la Statistique : Libreville, Juillet, 268 pages.

${ }^{3}$ Ministère de la planification, de l'environnement et du tourisme (1996), Réflexion stratégique à long terme, Gouvernement gabonais.

${ }^{4}$ Ben Hammouda, H., Oulmane, N. et Sadni Jallab, M. (2009), «D'une diversification spontanée à une diversification organisée. Quelles politiques pour diversifier les économies d'Afrique du Nord», Revue économique, 1 (60), 133-155.

Ben Hammouda, H. et coll. (2006), « La diversification, vers un nouveau paradigme pour le développement de l'Afrique ", CAPC Travail en Cours $N^{o} 36$, Commission Économique des Nations Unies pour l'Afrique, 165 pages. Kamgna, S.Y.(2007), "Diversification économique en Afrique Centrale: États des lieux et enseignements », Banque des États de l'Afrique Centrale, Munic Personal RePEc Archive: Online at http://mpra.ub.unimuenchen.de/9602/ MPRA Paper No. 9602, posted 16. July 2008 / 13:54

Markusen, J. R. and A. J. Venables (1999), «Foreign

Direct Investment as a Catalyst for Industrial

Development», European Economic Review (43), 335-56.

5 Dunning, J. H. (2001). The eclectic (OLI) paradigm of international production: past, present and future. International Journal of the Economics of Business, 8(2), 173-190.

Dunning, J. H. (1980). « Toward an eclectic theory of international production: Some empirical tests », Journal of International Business Studies 11(1): 9-31.

${ }^{6}$ Harding, T. \& Javorcik, B.S (2007), «Note on the effect of FDI on export diversification in Central and Eastern Europe», DECRG-TR

7 Dunning, J.H. (1998), «Location and Multinational

Enterprise: A Neglected Factor? », Journal of

International Business Studies, vol.29, no.1.

${ }^{8}$ CNUCED (2009), Rapport 2009 sur les Pays les Moins

Avancés : État, gouvernance et développement, Nations

Unies : New York et Genève

${ }^{9}$ Romer, P. [1990], Endogenous Technological Change, Journal of Political Economy, vol. 98, p. 71-102.

${ }^{10}$ Feenstra, R.C., Dorsanti, M, Tzu-Han, Y. and Chi-Yuan, L. (1999), «Testing endogenous growth in South Korea and Taiwan», Journal of Development Economics, vol. 60, pp. 317-341.
${ }^{11}$ Al-Marhubi F. [2000], Export diversification and growth: an empirical investigation, Applied Economic Letters, vol. 7, p. 559-562.

Berthélemy, J. C. et Söderling, L. (2001), «The Role of Capital Accumulation, Adjustment and Structural Change for Economic Take-Off: Empirical Evidence from African Growth Episodes ", World Development, vol. 29(2), p. 323-343.

Berthélemy, J. C. \& Chauvin, S. (2000), « Structural Changes in Asia and Growth Prospects after the Crisis », Document de travail du CEPII, no 00-09.

De Ferranti, D. et coll. (2002), « From Natural Resources to the Knowledge Economy Trade and Job quality», World Bank, Washington D.C.

${ }^{12}$ FMI (2009), Manuel de la balance des paiements et de la position extérieure globale, 6ème édition, Washington D.C.

${ }^{13}$ Barghouti, S., C. Timmer and P.R. Siegel (1990), «Rural diversification: Lessons from East Asia», World Bank Technical Paper \#I 17. Washington, DC: The World Rank. Petit, M., and S. Barghouti (1992), " Diversification: Challenges and opportunities » In Trends in agricultural diversification: Regional perspectives, edited by $\mathrm{S}$. Barghouti, L. Gaebus, and D. Umali. World Bank Technical Paper \#180. Washington, DC: The World Bank.

${ }^{14}$ Les personnes intéressées pour les détails de la méthode et des résultats de la régression linéaire multiple pourront contacter l'auteur à : paterne.ndjambou@uqac.ca 


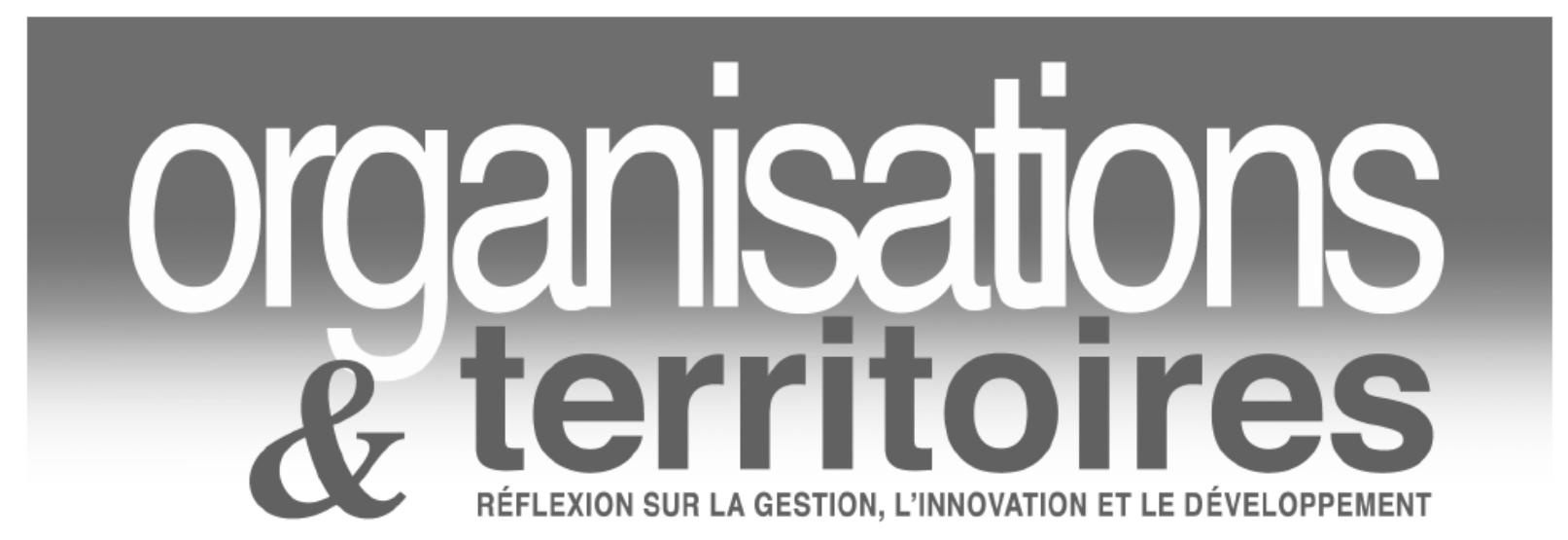

\section{Site Web}

\section{WWW.uqac.ca/revuleot}

www.uqac.ca/revueot

www.uqac.ca/revueot

www.uqac.ca/revueot

Nous vous invitons à l'explorer et à nous faire parvenir vos commentaires et suggestions afin d'en améliorer le contenu et la présentation. 